\title{
Relative Entropy and the Wigner-Yanase-Dyson-Lieb Concavity in an Interpolation Theory
}

\author{
A.Uhlmann \\ Department of Physics and NTZ, Karl-Marx-University, \\ DDR-701 Leipzig, German Democratic Republic
}

\begin{abstract}
We show that the Wigner-Yanase-Dyson-Lieb concavity is a general property of an interpolation theory which works between pairs of (hilbertian) seminorms. As an application, the theory extends the relevant work of Lieb and Araki to positive linear forms of arbitrary *-algebras. In this context a "relative entropy" is defined for every pair of positive linear forms of a *algebra with identity. For this generalized relative entropy its joint convexity and its decreasing under identity-preserving completely positive maps is proved.
\end{abstract}

\section{Introduction}

In this note we establish a generalization of the important Wigner-Yanase-Dyson conjecture that was proved by Lieb [1]. More precisely, we describe an interpolation procedure having the WYDL-concavity property. Essentially, the WYDLconcavity is the joint concavity in $A, B$ of

$$
A, B \rightarrow \operatorname{Tr}\left(X^{*} A^{1-t} X B^{t}\right), \quad 0<t<1,
$$

whenever (1) is well defined. The derivation of (1) at the value $t=0$ leads to the joint concavity of the expression

$$
A, B \rightarrow \operatorname{Tr}\left(X^{*} A X \ln B\right)-\operatorname{Tr}\left(X X^{*} A \ln A\right) .
$$

This functional reduces up to the sighn for $X=\mathbf{1}$ to the relative entropy of Lindblad [2], see also Umegaki [3]. From the concavity of (2) there is a short way to prove the strong subadditivity of entropy [4].

With the aid of the Tomita-Takesaki theory, Araki [5] was able to generalize most of the known properties of (1) and of the relative entropy to pairs of faithful normal states of $W^{*}$-algebras. In Araki's theory one has to rewrite (1) with the help of the relative modular operator of the two positive functionals

$$
Y \rightarrow \operatorname{Tr}(Y A) \text { and } Y \rightarrow \operatorname{Tr}(Y B)
$$


or, more generally, of two positive faithful normal states. However, in doing so and in deriving the desired inequalities one has to know deep results of the TomitaTakesaki theory and these results are not available for arbitrary *-algebras. We, therefore, substitute this theory by a certain interpolation technique in such a way, that (1) becomes the interpolation between the positive quadratic forms

$$
Y, X \rightarrow \operatorname{Tr}\left(A X Y^{*}\right) \quad \text { and } \quad Y, X \rightarrow \operatorname{Tr}\left(B Y^{*} X\right) .
$$

The generalized WYDL-inequalities will be expressed by the concavity in the positive hermitian forms (4) or, more generally, by the joint concavity of the interpolation with respect to a given pair of positive hermitian forms between which the interpolation takes place.

In defining an interpolation with a general WYDL-concavity we heavily use the notation and properties of the "geometrical mean" of Pusz and Woronowicz [6]. In handling positive hermitian forms and in deriving the desired inequalities, one may wonder wether there is not the possibility of extending some parts of the Tomita-Takesaki theory to more general *-algebras by our interpolation theory.

\section{Quadratical Means, Scales, and Interpolations of Seminorms}

Let us denote by $\mathscr{L}$ a complex linear space. Given two seminorms $p^{\prime}$ and $p^{\prime \prime}$ on $\mathscr{L}$, we define their "quadratical mean" $p$ as following: Denote by $S$ the set of all positive hermitian forms $\alpha$ satisfying

$$
|\alpha(x, y)| \leqq p^{\prime}(x) p^{\prime \prime}(y) \text { for all } x, y \in L .
$$

Then the quadratical mean $p$ is defined by

$$
p(x)=\sup _{\alpha \in S} \alpha(x, x)^{1 / 2} .
$$

Being uniquely determined by $p^{\prime}$ and $p^{\prime \prime}$ we shall write

$$
p=\mathrm{QM}\left(p^{\prime}, p^{\prime \prime}\right)
$$

to indicate that $p$ is the quadratical mean of these two seminorms.

Remark 1. It has been proved in Ref. [6] that $p$ is hilbertian for hilbertian $p^{\prime}, p^{\prime \prime}$ and the positive hermitian form associated with $p$ has been called "geometrical mean" by Pusz and Woronowicz.

Let $p, q$ be seminorms and $\lambda, \mu$ positive real numbers. Then

$$
\begin{aligned}
& \mathrm{QM}(p, q)=\mathrm{QM}(q, p), \quad \lambda \mu \mathrm{QM}(p, q)=\mathrm{QM}\left(\lambda^{2} p, \mu^{2} q\right), \\
& \mathrm{QM}(p, p)=p .
\end{aligned}
$$

Equation (8) are immediate consequences from the definition. If $f$ is a linear form and $|f| \leqq p$, then the hermitian form $x, y \rightarrow \overline{f(x)} f(y)$ can be used in the definition of $\mathrm{QM}(p, p)$. By the Hahn-Banach theorem we thus get $p \leqq \mathrm{QM}(p, p)$. The inverse inequality is a special case of the more general

$$
\mathrm{QM}(p, q)^{2}(x) \leqq p(x) q(x) \text {. }
$$


If $x=y$ in Equation (5), we find (10) as a consequence of (6).

The following proposition is evident.

Proposition 1. Let $\mathscr{L}_{0}$ be a subspace of $\mathscr{L}$ and denote by $p_{0}, q_{0}$ the restrictions of the seminorms $p, q$ onto $\mathscr{L}_{0}$. Then we have

$$
\mathrm{QM}\left(p_{0}, q_{0}\right)(x) \geqq \mathrm{QM}(p, q)(x) \text { for all } x \in \mathscr{L}_{0} .
$$

Proposition 2. Let us consider six seminorms $p, p_{1}, p_{2}, q, q_{1}, q_{2}$, and let $s_{1}, s_{2}$ be non-negative real numbers. If we have

$$
p^{2} \geqq s_{1}\left(p_{1}\right)^{2}+s_{2}\left(p_{2}\right)^{2} \quad \text { and } \quad q^{2} \geqq s_{1}\left(q_{1}\right)^{2}+s_{2}\left(q_{2}\right)^{2}
$$

it follows

$$
\mathrm{QM}(p, q)^{2} \geqq s_{1} \mathrm{QM}\left(p_{1}, q_{1}\right)^{2}+s_{2} \operatorname{QM}\left(p_{2}, q_{2}\right)^{2} .
$$

The importance of this proposition is in its "self-reproducing" character. The proof can be given by a simple modification of the corresponding one in [6]. One can, however, prove a more general statement and this we are going to do. Let us mention first, that we can restrict ourselves to the case $s_{1}=s_{2}=1$ by virtue of Equation (8).

A real valued function $\phi$ defined on $R^{n}$ is called a monotonous one if

$$
\phi(\xi) \geqq \phi(\eta) \quad \text { for } \quad\left|\xi_{i}\right| \geqq\left|\eta_{i}\right|, \quad i=1, \ldots, n .
$$

Let $\phi$ be a monotonous seminorm on $R^{n}$ and assume $p_{1}, \ldots, p_{n}$ to be seminorms on $\mathscr{L}$. Then $\phi\left(p_{1}, \ldots, p_{n}\right)$ is a seminorm on $\mathscr{L}$.

Proposition 3. Let $p_{1}, \ldots, p_{n}, q_{1}, \ldots, q_{n}$ be seminorms on the linear space $\mathscr{L}$. Let further $\phi, \psi$ be two monotonous seminorms on $R^{n}$ with the property

$$
\phi(\xi) \psi(\eta) \geqq\left|\sum_{j} \xi_{j} \eta_{i}\right|
$$

for all $\xi, \eta \in R^{n}$. Then from

$$
p \geqq \phi\left(p_{1}, \ldots, p_{n}\right), \quad q \geqq \psi\left(q_{1}, \ldots, q_{n}\right)
$$

it follows for the seminorms $p, q$ the inequality

$$
\mathrm{QM}(p, q)^{2} \geqq \sum \mathrm{QM}\left(p_{j}, q_{j}\right)^{2} .
$$

Proof. For all $x, y \in \mathscr{L}$ we substitute in (14)

$$
\xi_{i}=p_{i}(x), \quad \eta_{j}=q_{j}(y) .
$$

We denote by $S_{j}$ the set of all positive hermitian forms $\alpha$ satisfying $|\alpha(x, y)| \leqq p_{i}(x) q_{i}(y)$. Equations (15) and (14) imply

$$
p(x) q(y) \geqq \sum p_{j}(x) q_{j}(y) \geqq\left|\sum \alpha_{j}(x, y)\right| .
$$

Therefore,

$$
\mathrm{QM}(p, q)(x) \geqq\left\{\sum \alpha_{j}(x, x)\right\}^{1 / 2} .
$$

Taking the suprema over the sets $S_{j}$ we get (16).

Applying the Schwarz inequality to Proposition 3 one gets Proposition 2. 
Let us now consider some examples, the first of which is due to Woronowicz and the others are consequences of the first.

Example 1. Let $\mathscr{L}$ be a hilbert space with scalar product (., .). Assume $A_{j} \in \mathscr{B}_{+}(\mathscr{L})$ and $A_{1} A_{2}=A_{2} A_{1}$. Then the quadratical mean of the two seminorms $x \rightarrow\left(x, A_{j} x\right)^{1 / 2}$ is equal to the seminorm $x \rightarrow\left(x,\left(A_{1} A_{2}\right)^{1 / 2} x\right)^{1 / 2}$ (see Ref. [6]).

Example 2. Let us calculate the quadratical mean of the seminorms associated with the positive hermitian forms (4). Using the hilbert space of the HilbertSchmidt operators with scalar product $Y, X \rightarrow \operatorname{Tr}\left(Y^{*} X\right)$, Example 1 immediately allows the conclusion that their quadratical mean is given by the square root of the right hand side of (1) with $t=1 / 2$. We mention that Proposition 2 gives the Yanase-Wigner concavity.

Example 3. Let us return to Example 1 again and let us now treat the case $A_{1} A_{2} \neq A_{2} A_{1}$ but under the assumption of the existence of $\left(A_{1}+A_{2}\right)^{-1}$ in $\mathscr{B}(\mathscr{L})$. Then the quadratical mean is given by $x \rightarrow(x, C x)^{1 / 2}$ with $C \geqq 0$ and

$$
\begin{aligned}
C & =\left(A_{1}+A_{2}\right)^{1 / 2}\left(B_{1} B_{2}\right)^{1 / 2}\left(A_{1}+A_{2}\right)^{1 / 2}, \\
B_{j} & =\left(A_{1}+A_{2}\right)^{-1 / 2} A_{j}\left(A_{1}+A_{2}\right)^{-1 / 2} .
\end{aligned}
$$

This, indeed, is nothing but the calculation of Pusz and Woronowicz: $B_{1}$ and $B_{2}$ commute and $x \rightarrow\left(A_{1}+A_{2}\right)^{1 / 2} x$ maps the hilbert space onto itself. The general case can be obtained by a limiting procedure [6].

After these preliminarities we come to interpolation theory in the spirit of Krein and Calderón [7]. Indeed, we generalize some concepts of interpolation theory as given in the exposition of Palais [8]. However, the concave dependence on the "boundary data" of interpolations seemingly was not examined up to now.

Definition 1. Let $t^{\prime}<t^{\prime \prime}$ be two reals, possibly infinite, and let $L$ be a complexlinear space. A map

$$
t \rightarrow p_{t}, \quad t \in\left[t^{\prime}, t^{\prime \prime}\right]
$$

which associates to every real number of the given interval a seminorm of $\mathscr{L}$ is called a "quadratical scale" on $\left[t^{\prime}, t\right.$ " $]$ if for every $x \in \mathscr{L}$ the function $t \rightarrow p_{t}(x)$ is continuous and if

$$
p_{t}=\mathrm{QM}\left(p_{t_{1}}, p_{t_{2}}\right) \text { for all } 2 t=t_{1}+t_{2}
$$

with $t_{1}, t_{2} \in\left[t^{\prime}, t^{\prime \prime}\right]$ is valid.

A bit more involved is the notation of quadratical interpolation, for here we have to relaxe in a suitable way the continuity at the boundary of the interval in question. Later on we shall only consider interpolations: To formulate the corresponding results for scales is a simple rewriting.

Definition 2. Let $p^{\prime}, p^{\prime \prime}$ be two seminorms of the complex-linear space $\mathscr{L}$. A "quadratical interpolation" from $p^{\prime}$ to $p^{\prime \prime}$ is a quadratical scale $t \rightarrow p_{t}$ on $[0,1]$ with the following three properties:

$$
\begin{aligned}
p_{1 / 2} & =\mathrm{QM}\left(p^{\prime}, p^{\prime \prime}\right), \\
p_{t / 2} & =\mathrm{QM}\left(p^{\prime}, p_{t}\right), \quad t \in[0,1], \\
p_{(1+t) / 2} & =\mathrm{QM}\left(p^{\prime \prime}, p_{t}\right), \quad t \in[0,1] .
\end{aligned}
$$


Proposition 4. For a given pair $p^{\prime}, p^{\prime \prime}$ of seminorms there exists at most one quadratical interpolation from $p^{\prime}$ to $p^{\prime \prime}$.

The proof of this uniqueness theorem is typical for the proofs of the following assertions too. Let $T$ be the set of reals $t$ for which all interpolations from $p^{\prime}$ to $p^{\prime \prime}$ coincide. Because of the continuity of scales (Definition 1) the set $T$ is closed. (21) tells us $1 / 2 \in T$. (22) and (23) show that with $t \in T$ also $t / 2 \in T$ and $(1+t) / 2 \in T$. Hence $0 \in T$ and $1 \in T$ for $T$ is closed. Equation (20) indicates $\left(t_{1}+t_{2}\right) / 2 \in T$, whenever $t_{1}, t_{2} \in T$. A closed set with this property is a convex one. Such a set, containing $t=0,1$, contains the whole interval $[0,1]$. Therefore, all quadratical interpolations from $p^{\prime}$ to $p^{\prime \prime}$ coincide.

Notation. By virtue of Proposition 4 the quadratical interpolation, if it exists, is defined by $p^{\prime}$ and $p^{\prime \prime}$ uniquely. We write

$$
p_{t}=\mathrm{QI}_{t}\left(p^{\prime}, p^{\prime \prime}\right)
$$

if $t \rightarrow p_{t}$ is the quadratical interpolation from $p^{\prime}$ to $p^{\prime \prime}$. In writing (24) we implicitely assume the existence of the interpolation.

Proposition 5. If for $x \in \mathscr{L}$ fixed and for one $t_{0} \in[0,1]$ we have $\mathrm{QI}_{t_{0}}\left(p^{\prime}, p^{\prime \prime}\right)(x)=0$, then $\mathrm{QI}_{t}\left(p^{\prime}, p^{\prime \prime}\right)(x)=0$ for all $t \in[0,1]$.

Proof. Using Equation (10) we find with the argument of the proof of Proposition 4 the validity of Proposition 5. Namely, the set of all $t$ for which $\mathrm{QI}_{t}\left(p^{\prime}, p^{\prime \prime}\right)(x)=0$ is a closed convex set. This set is either empty or it contains $t=0$ and $t=1$.

Proposition 6. Suppose $p_{t}=\mathrm{QI}_{t}\left(p^{\prime}, p^{\prime \prime}\right)$. Then for all numbers $t_{1}, \ldots, t_{m}, s_{1}, \ldots, s_{m}$, $t \in[0,1]$ with $s_{1}+\ldots+s_{m}=1$ we have

$$
\begin{aligned}
p_{\left(s_{1} t_{1}+\ldots+s_{m} t_{m}\right)} & \leqq \prod_{j=1}^{m}\left(p_{t_{j}}\right)^{s_{J}}, \\
p_{t} & \leqq\left(p^{\prime}\right)^{1-t}\left(p^{\prime \prime}\right)^{t} .
\end{aligned}
$$

Proof. To prove (25) we may (according to Proposition 5) assume $p_{t}(x) \neq 0$. The function $t \rightarrow f(t)=\ln p_{t}(x)$ is continuous. Now, $p_{t}$ is a scale and we may apply Equation (10) to show $f\left(t_{1} / 2+t_{2} / 2\right) \leqq 1 / 2 f\left(t_{1}\right)+1 / 2 f\left(t_{2}\right)$. By continuity, $f$ is a convex function, therefore. Hence $f$ satisfies the inequality of Jensen and thus (25) is valid. To prove (26), we find firstly the set $T$ of all $t$ for which (20) is true to be closed. Applying (10) we easily find $T$ convex, containing with $t$ also $t / 2$ and $(1+t) / 2$ and, last not least, the number $t=1 / 2$. Therefore, $T=[0,1]$.

The Proposition 6 is in its form a standart one in interpolation theory. More important for us is, however, the consequences of Proposition 2 for the quadratical interpolation. Before writing down this consequence we should mention a notable inequality.

\section{Proposition 7. (Monotonicity).}

$$
\mathrm{QI}_{t}\left(p^{\prime}, p^{\prime \prime}\right) \geqq \mathrm{QI}_{t}\left(q^{\prime}, q^{\prime \prime}\right) \quad \text { if } \quad p^{\prime} \geqq q^{\prime} \quad \text { and } \quad p^{\prime \prime} \geqq q^{\prime \prime} \text {. }
$$

Proof. From the very definition of the quadratical mean we see the validity of (27) if we replace $\mathrm{QI}_{t}$ by the functor QM. With the help of this we conclude by our 
standart argument that (27) should be valid for a closed convex set of numbers $t$ including $t=0$ and $t=1$ and hence the whole interval $[0,1]$.

Proposition 8. (Lieb Concavity). Assume $p^{\prime}, p^{\prime \prime}, q^{\prime}, q^{\prime \prime}, r^{\prime}, r^{\prime \prime}$ to be seminorms on the complex-linear space $\mathscr{L}$. If for a number $s$ with $0 \leqq s \leqq 1$ the inequalities

$$
\begin{gathered}
\left(r^{\prime}\right)^{2} \geqq s\left(p^{\prime}\right)^{2}+(1-s)\left(q^{\prime}\right)^{2} \\
\left(r^{\prime \prime}\right)^{2} \geqq s\left(p^{\prime \prime}\right)^{2}+(1-s)\left(q^{\prime \prime}\right)^{2}
\end{gathered}
$$

are valid, then for all $t \in[0,1]$ it is

$$
\mathrm{QI}_{t}\left(r^{\prime}, r^{\prime \prime}\right)^{2} \geqq s \mathrm{QI}_{t}\left(p^{\prime}, p^{\prime \prime}\right)^{2}+(1-s) \mathrm{QI}_{t}\left(q^{\prime}, q^{\prime \prime}\right)^{2}
$$

Proof. Here we consider the closed set of all $t$ for which (29) is valid. By Proposition 2 this set $T$ is convex, for a quadratical interpolation is a scale. Applying Proposition 2 to the Equations (21)-(23) one sees $T=[0,1]$.

As Example 5 below shows, Proposition 8 is a generalization of the WignerYanase-Dyson-Lieb concavity not using the concept of trace nor of relative modular operator.

Lastly, we consider two complex-linear spaces $\mathscr{L}$ and $\mathscr{L}^{\prime}$ and a linear map $\Phi$ from $\mathscr{L}^{\prime}$ into $\mathscr{L}$. Denoting by $\Phi^{+}$the adjoint mapping, every seminorm $p$ gives rise to a seminorm $p^{\prime}=\Phi^{+} p$ of $\mathscr{L}^{\prime}$ by the definition $p^{\prime}\left(x^{\prime}\right)=p\left(\Phi x^{\prime}\right)$ with $x^{\prime} \in \mathscr{L}^{\prime}$. Every inequality of the form $|\alpha(x, y)| \leqq p(x) q(y)$ on $\mathscr{L}$ implies the corresponding inequality on $\mathscr{L}^{\prime}$ by the substitution $x=\Phi x^{\prime}, y=\Phi y^{\prime}$. Therefore, QM $\left(p^{\prime}, q^{\prime}\right)\left(x^{\prime}\right)$ cannot be less than QM $(p, q)\left(\Phi x^{\prime}\right)$. This proves

$$
\mathrm{QM}(p, q)\left(\Phi x^{\prime}\right) \leqq \mathrm{QM}\left(\Phi^{+} p, \Phi^{+} q\right)\left(x^{\prime}\right), \quad x^{\prime} \in \mathscr{L}^{\prime} .
$$

Now we can play our standart game and replace QM by $\mathrm{QI}_{t}$, provided, as usual, the interpolations exist. Thus we get

Proposition 9. Let $\Phi$ be a linear map from $\mathscr{L}$ into $\mathscr{L}^{\prime}$. For every pair of seminorms $p, q$ on $\mathscr{L}$ and for all $t \in[0,1]$ it holds the inequality

$$
\mathrm{QI}_{t}(p, q)\left(\Phi x^{\prime}\right) \leqq \mathrm{QI}_{t}\left(\Phi^{+} p, \Phi^{+} q\right)\left(x^{\prime}\right), \quad x^{\prime} \in L^{\prime} .
$$

Note that in (31) we have to assume the existence of both interpolations.

Remark 2. a) Every quadratical interpolation is a quadratical scale. b) Let $t \rightarrow p_{t}$ be a scale. Replacing $t$ by a linear transformation of $t$ we get a new scale. c) The restriction onto a subintervall of a quadratical scale is a quadratical scale again. d) Applying these trivial statements, the Propositions 4-9 can be converted into properties of scales.

Remark 3. If we have $\mathscr{L}=\mathscr{L}^{\prime}$ in Proposition 9 and furthermore

$$
\Phi^{+} q \leqq q, \quad \Phi^{+} p \leqq p
$$

we may combine (31) with Proposition 7. The obvious result

$$
\mathrm{QI}_{t}(p, q)(\Phi x) \leqq \mathrm{QI}_{t}(p, q)(x), \quad 0 \leqq t \leqq 1,
$$

is an interpolation theorem a la Riesz-Thorin [9]. 
Remark 4. The Equations (8) and (9) imply $\mathrm{QI}_{t}(p, q)=\mathrm{QI}_{1-t}(q, p), \mathrm{QI}_{t}(p, p)=p$, and $\mathrm{QI}_{t}(\lambda p, \mu q)=\lambda^{1-t} \mu^{t} \mathrm{QI}_{t}(p, q)$.

Example 4. Assuming the same situation as in Example 1, the quadratical interpolation from $x \rightarrow\left(x, A_{1} x\right)^{1 / 2}$ to $x \rightarrow\left(x, A_{2} x\right)^{1 / 2}$ is given by

$$
p_{t}(x)=\left(x, A_{1}^{1-t} A_{2}^{t} x\right)^{1 / 2} \text { for } 0 \leqq t \leqq 1,
$$

$p_{0}$ and $p_{1}$ are given by the limits $t \rightarrow 0$ and $t \rightarrow 1$ in Equation (34). As an important consequence, $\left(p_{t}\right)^{2}(x)$ can be extended analytically to an holomorphic function in the strip $0<\operatorname{Re} t<1$ and to a continuous function in $0 \leqq \operatorname{Re} t \leqq 1$.

Example 5. Consider again Example 2. With respect to the scalar product $X, Y \rightarrow \operatorname{Tr}\left(X^{*} Y\right)$ the mappings $Y \rightarrow A Y$ and $Y \rightarrow Y B$ with positive $A, B$ are positive mappings which commute. Hence the formula of Example 4 can be used to compute the quadratical interpolation between the positive hermitian forms (4). A simple calculation shows that the interpolation is given by the seminorms associated with (1). Therefore, from Proposition 8 the WYDL inequalities follow.

Example 6. In the situation of Example 3 the corresponding quadratical interpolation is given by

with

$$
x \rightarrow p_{t}(x)=\left(x, C_{t} x\right)^{1 / 2}, \quad 0<t<1,
$$

$$
C_{t}=\left(A_{1}+A_{2}\right)^{1 / 2} B_{1}^{1-t} B_{2}^{t}\left(A_{1}+A_{2}\right)^{1 / 2},
$$

where $B_{1}, B_{2}$ is defined by Equation (18). Going in (35a) to the limits $t \rightarrow 0,1$ one obtains the complete interpolation. Proposition 8 shows that for all $0<t<1$ the map

$$
A_{1}, A_{2} \rightarrow C_{t}
$$

is jointly operator concave in $A_{1}, A_{2}$. This is an apparently stronger statement than the operator concavity of $A \rightarrow A^{t}$ for $0<t<1$, see [10].

In the case of the bounded existence of $A_{1}^{-1}$ it is possible to rewrite (35) in the following form

$$
\begin{aligned}
C_{t} & =A_{1}\left(A_{1}+A_{2}\right)^{-1 / 2} D^{t}\left(A_{1}+A_{2}\right)^{1 / 2}, \\
D & =\left(A_{1}+A_{2}\right)^{1 / 2}\left(A_{1}\right)^{-1}\left(A_{2}\right)\left(A_{1}+A_{2}\right)^{-1 / 2} .
\end{aligned}
$$

One easily sees $D=D^{*} \geqq 0$. The representation indicates some connections with the theory of semigroups: $t \rightarrow\left(A_{1}\right)^{-1} C_{t}$ can be defined naturally for $0 \leqq t<\infty$ and is a semigroup. Under this circumstances, $t \rightarrow p_{t}$ is a scale on $(0, \infty)$ and $p_{1} \leftrightarrow A_{2}$.

\section{Interpolation of Positive Hermitian Forms}

We are now in the position to state the existence of the quadratical interpolation in the case of hermitian seminorms.

Proposition 10. Let $\mathscr{L}$ be a complex-linear space. For every pair of positive hermitian forms $\alpha, \beta$ there exists one and only one function

$$
z \rightarrow \gamma_{z}, \quad 0 \leqq \operatorname{Re} z \leqq 1
$$


with values in the set of sesquilinear forms on $\mathscr{L}$ with the following properties:

(i) The function $z \rightarrow \gamma_{z}(x, y)$ is continuous in $0 \leqq \operatorname{Re} z \leqq 1$ and holomorphic in $0<\operatorname{Re} z<1$ for all $x, y \in L$.

(ii) $\gamma_{t}$ is a positive hermitian form for $0 \leqq t \leqq 1$ and

$t \rightarrow p_{t}$ with $p_{t}(x)=\gamma_{t}(x, x)^{1 / 2}$

is the quadratical interpolation from $x \rightarrow \alpha(x, x)^{1 / 2}$ to $x \rightarrow \beta(x, x)^{1 / 2}$.

(iii) It is

$\bar{\gamma}_{z}=\gamma_{\bar{z}}$

and with $\operatorname{Re} z=\left(t_{1}+t_{2}\right) / 2$ one has

$\left|\gamma_{z}(x, y)\right| \leqq p_{t_{1}}(x) p_{t_{2}}(y)$.

(iv) For every $0 \leqq t \leqq 1$ the map

$\alpha, \beta \rightarrow \gamma_{t}$

is jointly concave in $\alpha$ and $\beta$.

Proof. We construct the hilbert space associated canonically with the positive hermitian form $\alpha+\beta$. This transfers the problem to the situation described by Examples 4 and 1.

Example 4 explicitely shows the existence of the quadratical interpolation ii) and the existence of the analytic continuation i). From the explicit expression (34) we easily find iii). iv) is a reformulation of Proposition 8 . The uniqueness of the construction is to be seen from Proposition 4.

Remark 5. In the sense of the functional calculus of forms introduced by Woronowicz and Pusz [6] one can write with the aid of their symbolic notation

$$
\gamma_{t}=(\alpha)^{1-t}(\beta)^{t} \quad \text { if } \quad 0<t<1 .
$$

We prefer, however, another notation.

Notation. In the situation described by Proposition 10 we write

$$
\gamma_{z}=\mathrm{QF}_{z}(\alpha, \beta)
$$

Especially, if $p, q$ denote the seminorms defined by the positive hermitian forms $\alpha, \beta$ we have for $t \in[0,1]$

$$
\mathrm{QI}_{t}(p, q)^{2}(x)=\mathrm{QF}_{t}(\alpha, \beta)(x, x) \text {. }
$$

\section{The Relative Entropy Functional}

In the following we restrict ourselves to hermitian seminorms, though the general case of pairs of seminorms can be handled as well.

Definition 3. For every pair $\alpha, \beta$ of positive hermitian forms on the complex-linear space $\mathscr{L}$ and for all $x \in \mathscr{L}$ we define the functional

$$
S(\alpha ; \beta): x \rightarrow S(\alpha ; \beta)(x)
$$


by

$$
S(\alpha ; \beta)(x)=-\liminf _{t \rightarrow+0} \frac{\mathrm{QF}_{t}(\alpha, \beta)(x, x)-\alpha(x, x)}{t} .
$$

$S(\alpha ; \beta)$ is called "relative entropy functional of the pair $\alpha, \beta$ ".

Proposition 11. (Convexity). The relative entropy functional is jointly convex in the pair $\alpha, \beta$.

Proof. Proposition 10 guarantees the joint concavity of $\alpha, \beta \rightarrow \mathrm{QF}_{t}(\alpha, \beta)-\alpha$. The lower limit of concave functions is concave again. Because of the minus sighn, (45) defines a convex function of the pair $\alpha, \beta$.

Proposition 12.(Peierls-Bogoliubov Inequality).

$$
S(\alpha ; \beta)(x) \geqq \alpha(x, x)\{\ln \alpha(x, x)-\ln \beta(x, x)\} .
$$

Proof. Remembering all the definitions, the inequality is an estimate of (45) with the help of Proposition 6, Equation (26).

Applying Klein's inequality to the right hand side of (46) one gets

$$
S(\alpha ; \beta)(x) \geqq \alpha(x, x)-\beta(x, x) .
$$

Proposition 13. Let $\Phi$ be a linear map from $\mathscr{L}^{\prime}$ into $\mathscr{L}$ and denote $\Phi$ the transposed map $(\tilde{\phi} \alpha)\left(x^{\prime}, y^{\prime}\right)=\alpha\left(\Phi x^{\prime}, \Phi y^{\prime}\right)$. For every pair $\alpha, \beta$ of positive hermitian forms defined on $\mathscr{L}$ we have for all $x^{\prime} \in \mathscr{L}^{\prime}$

$$
S(\alpha ; \beta)\left(\Phi x^{\prime}\right) \geqq S(\tilde{\Phi} \alpha ; \tilde{\Phi} \beta)\left(x^{\prime}\right) .
$$

Proof. Due to (45) this inequality is an immediate consequence of Proposition 9, Equation (31).

Applying (48) to the situation of a subspace $\mathscr{L}_{0} \subseteq \mathscr{L}$ and to the imbedding map $\mathscr{L}_{0} \rightarrow \mathscr{L}$, we get the important

Proposition 14. (Monotonicity). Let $\alpha, \beta$ be a pair of positive hermitian forms on $\mathscr{L}$ and denote by $\alpha_{0}, \beta_{0}$ their restrictions onto the subspace $\mathscr{L}_{0}$ of $\mathscr{L}$. Then for all $x_{0} \in \mathscr{L}_{0}$

$$
S(\alpha ; \beta)\left(x_{0}\right) \geqq S\left(\alpha_{0} ; \beta_{0}\right)\left(x_{0}\right) .
$$

Next we mention a consequence of Proposition 7. For fixed $\alpha$ the functor $\mathrm{QF}_{t}$ is monotonous in $\beta$ if $t$ is in the unit interval. Using this in the defining Equation (45) we obtain

Proposition 15. Give three positive hermitian forms $\alpha, \beta, \beta^{\prime}$ on $\mathscr{L}$, one has

$$
S(\alpha ; \beta) \leqq S\left(\alpha ; \beta^{\prime}\right) \text { if } \beta \geqq \beta^{\prime} .
$$

Remark 6. If with some positive numbers $\lambda$, $\mu$ we have $\lambda \alpha \leqq \beta \leqq \mu \alpha$ one can express the relative entropy functional with the help of the Woronowicz-Pusz functional calculus (see Remark 5) by the symbolic formula

$$
S(\alpha ; \beta)=\alpha(\ln \alpha-\ln \beta) \text {. }
$$




\section{Quadratical Interpolations in *-Algebras}

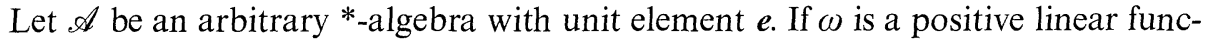
tional on $\mathscr{A}$, we define for all $\boldsymbol{a}, \boldsymbol{b} \in \mathscr{A}$ the positive hermitian forms

$$
\begin{aligned}
\omega^{L}(\boldsymbol{a}, \boldsymbol{b}) & =\omega\left(\boldsymbol{a}^{*} \boldsymbol{b}\right) \\
\omega^{R}(\boldsymbol{a}, \boldsymbol{b}) & =\omega\left(\boldsymbol{b} \boldsymbol{a}^{*}\right) .
\end{aligned}
$$

Remark 7. If we assume in Equation (3) $A, B$ to be trace class operators, then (3) defines two normal positive linear functional $\omega$ and $v$ of the *-algebra of all bounded operators of a certain hilbert space. Now we can identify the two positive hermitian forms (4) with $\omega^{R}$ and $v^{L}$.

The remark above and Example 5 again show that the WYDL-concavity is contained in the following statement.

Proposition 16. For every $\boldsymbol{a} \in \mathscr{A}$ and all $0 \leqq t \leqq 1$ the map

$$
\omega, v \rightarrow \mathrm{QF}_{t}\left(\omega^{R}, v^{L}\right)(\boldsymbol{a}, \boldsymbol{a})
$$

is jointly concave in $\omega, v$.

It is clear that this proposition is a rewriting of Proposition 8 or 10 . We do not reformulate the other relevant propositions in terms of positive linear forms. It is completely evident how to do this. We add the connection with the relative entropy.

Definition 4. Let $\omega, v$ be two positive linear functionals of the *-algebra $\mathscr{A}$ with unit element $e$. Their relative entropy $S(\omega / v)$ is defined by

$$
S(\omega / v)=S\left(\omega^{R} ; v^{L}\right)(e) .
$$

Again we find it unnecessary to write down explicitly the direct implications of Propositions 11-15.

However, there is a non-trivial point concerning completely positive maps. We do not need the full content [11] of this concept. That is to be seen below.

Proposition 17. Let $\mathscr{A}, \mathscr{B}$ be two $*$-algebras and $\Phi$ a linear map from $\mathscr{A}$ into $\mathscr{B}$ satisfying

$$
\begin{aligned}
& \Phi\left(a^{*}\right)=\Phi(a)^{*}, \quad a \in \mathscr{A}, \\
& \Phi(a)^{*} \Phi(a) \leqq \Phi\left(a^{*} a\right) .
\end{aligned}
$$

If $\omega$ is a positive linear form of $\mathscr{B}$, then $\omega_{\Phi}$ defined by

$$
\omega \phi(\boldsymbol{a})=\omega(\Phi \boldsymbol{a})
$$

is a positive linear form of $\mathscr{A}$. For any two positive linear forms $\omega, v$ of $\mathscr{B}$ we have the inequalities, $0 \leqq t \leqq 1$,

$$
\mathrm{QF}_{t}\left(\omega^{R}, v^{L}\right)(\Phi \boldsymbol{a}, \Phi \boldsymbol{a}) \leqq \mathrm{QF}_{t}\left(\omega_{\Phi}^{R}, v_{\Phi}^{R}\right)(\boldsymbol{a}, \boldsymbol{a}) .
$$

Remark 8. (54) and (55) imply the positivity of $\Phi$ and hence (56) defines a positive linear form of $\mathscr{A}$. On the other hand it is well known for $C^{*}$-algebras that complete positivity and $e_{\mathscr{B}} \geqq \phi\left(e_{\mathscr{A}}\right)$ imply $(55)$. 
Proof. Write

$$
p(\boldsymbol{b})=\omega\left(\boldsymbol{b} \boldsymbol{b}^{*}\right)^{1 / 2}, \quad q(\boldsymbol{b})=v\left(\boldsymbol{b}^{*} \boldsymbol{b}\right)^{1 / 2}
$$

and

$$
\tilde{p}(a)=p(\Phi \boldsymbol{a}), \quad \tilde{q}(a)=q(\Phi \boldsymbol{a})
$$

with $\boldsymbol{a} \in \mathscr{A}$ and $\boldsymbol{b} \in \mathscr{B}$. According to (31) we have

$$
\mathrm{QF}_{t}\left(\omega^{R}, \omega^{L}\right)(\Phi \boldsymbol{a}, \Phi \boldsymbol{a})=\mathrm{QI}_{t}(p, q)(\Phi \boldsymbol{a})^{2} \leqq \mathrm{QI}_{t}(\tilde{p}, \tilde{q})(\boldsymbol{a})^{2}
$$

Next we have according to (54) and (55)

$$
\omega_{\Phi}^{R}(\boldsymbol{a}, \boldsymbol{a})=\omega_{\Phi}\left(\boldsymbol{a} \boldsymbol{a}^{*}\right)=\omega\left(\Phi\left(\boldsymbol{a} \boldsymbol{a}^{*}\right)\right) \geqq \omega\left((\Phi \boldsymbol{a})(\Phi \boldsymbol{a})^{*}\right)=\tilde{p}(\boldsymbol{a})^{2} .
$$

The same reasoning gives

$$
v_{\Phi}^{L}(\boldsymbol{a}, \boldsymbol{a}) \geqq q(\Phi \boldsymbol{a})^{2}=\tilde{q}(\boldsymbol{a})^{2} .
$$

If we now combine the inequalities (59) with Proposition 7 we obtain

$$
\mathrm{QI}_{t}(\tilde{p}, \tilde{q})(\boldsymbol{a})^{2} \leqq \mathrm{QF}_{t}\left(\omega_{\Phi}^{R}, v_{\Phi}^{L}\right)(\boldsymbol{a}, \boldsymbol{a}) \text {. }
$$

Putting together (58) and (60) we obtain (57).

It follows from the proof that we can replace in $(57)$ the letter " $L$ " by the letter " $R$ " (for example) and the inequality remains true.

To derive from Proposition 17 an entropy inequality, one has to insure

$$
\omega^{R}(\Phi e, \Phi e)=\omega_{\Phi}^{R}(e, e) .
$$

This is true if $\phi$ maps the unit of $\mathscr{A}$ on the unit of $\mathscr{B}$, i.e.:

Proposition 18. If under the same assumption as in Proposition 17 we have $\Phi \boldsymbol{e}_{\mathscr{A}}=\boldsymbol{e}_{\mathscr{B}}$, where $\boldsymbol{e}_{\mathscr{A}}$ and $\boldsymbol{e}_{\mathscr{B}}$ denote the unit elements of $\mathscr{A}$ and $\mathscr{B}$ respectively, then

$$
S(\omega / v) \geqq S\left(\omega_{\Phi} / v_{\Phi}\right) \text {. }
$$

This generalize a theorem of Lindblad [2].

Let us consider a further question. Let us replace in (52) the form $v^{L}$ by $v^{R}$. Going to the GNS-representation of $\omega+v$ we meet the situation of Examples 1 and 4 with $A_{1}, A_{2}$ in the weak commutator of this representation. If the weak commutator is a von Neumann algebra, then it contains $A_{1}^{1-t} A_{2}^{t}$ and the corresponding positive hermitian form equals $\mu^{R}$ with a certain positive linear form $\mu$. This is already the proof of

Proposition 19. Let $\omega, v$ be two positive linear forms of the *algebra $\mathscr{A}$. If the weak commutator of the GNS-representation of $\omega+v$ is a von Neumann algebra, then there exists a map

$$
t \rightarrow \mu_{t}, \quad 0 \leqq t \leqq 1,
$$

into the positive linear functionals of $A$ such that

$$
\left(\mu_{t}\right)^{R}=\mathrm{QF}_{t}\left(\omega^{R}, v^{R}\right) .
$$

Acknowledgements. I am grateful to S. L. Woronowicz, Warsaw, for introducing me into his theory prior to publication. I thank A. Pietsch, H. Triebel, Jena, and G. Lassner, Leipzig, for discussions. 


\section{References}

1. Lieb,E.H.: Advan. Math. 11, 267 (1973)

2. Lindblad, G.: Commun. math. Phys. 39, 111 (1974)

3. Umegaki, H.: Kodai Math. Sem. Rep. 14, 59 (1962)

4. Lieb,E.H., Ruskai, M.B.: Phys. Letters 30, 434 (1973); J. Math. Phys. 14, 1938 (1973)

5. Araki,H.: RIMS-188, RIMS-190, RIMS-191, Kyoto 1975

6. Pusz, W., Woronowicz, S.L.: Rep. Math. Phys. 8, 159 (1975)

7. Krein, S. G.: Dokl. Akad. Nauk. 130, 491 (1960); 132, 510 (1960); Calderón,A. P.: Studia Math. 24, 113 (1964)

8. Palais, R. S.: Seminar on the Atiyah-Singer index theorem, Chapter VIII. Princeton: University Press 1965

9. Hewitt,E., Ross, K.A.: Abstract harmonic analysis. II. Berlin-Heidelberg-New York: Springer 1970

10. Kraus, F.: Math. Z. 41, 18 (1936)

Bendat, J., Sherman, S.: Trans. Amer. Math. Soc. 79, 58 (1955)

11. Stinespring, W.F.: Proc. Amer. Math. Soc. 6, 211 (1955)

Umegaki, H.: Tôhoku Math. J. 7, 206 (1955)

Communicated by H. Araki

Received September 10, 1976 